\title{
Visual Data Analysis of Production Quality Data for Aluminum Casting
}

\author{
Nikolina Jekic \\ Pro2Future GmbH Graz, Austria \\ nikolina.jekic@pro2future.at \\ Belgin Mutlu \\ Pro2Future GmbH Graz, Austria \\ Know-Center GmbH Graz, Austria \\ belgin.mutlu@pro2future.at
}

\author{
Manuela Schreyer \\ Austria Metall GmbH, Austria \\ manuela.schreyer@amag.at
}

Tobias Schreck

Graz University of Technology, Austria

tobias.schreck@cgv.tugraz.at

\author{
Steffen Neubert \\ AMAG casting $\mathrm{GmbH}$, Austria \\ steffen.neubert@amag.at
}

\begin{abstract}
Today's manufacturing industry is shaped by the Industry 4.0 vision, which is to increase the number of individual goods produced while minimizing the production costs and time. To increase the production outcome and quality, users need to continuously monitor and adjust the entire process. While the recent advances in sensor technology can help users to collect, produce and exchange data, human beings are often overwhelmed by the amount of data being collected. Still, the human visual system is a powerful tool that can be used to decode and process large datasets. To make intelligent use of this ability, we have developed an interactive visual data analysis tool called ADAM that can support production data exploration in the aluminum industry. Furthermore, we demonstrate the effectiveness of our tool using real production data and present insights which could be gained from use of our tool by domain experts.
\end{abstract}

\section{Introduction}

The demand for high-quality material in aluminum products has constantly increased in recent years. Therefore, researchers need to develop new methods and tools to support material engineers, enabling them to continuously monitor, optimize, and improve their production processes. To meet a key criterion in quality assessment, these engineers strive to minimize or avoid indications e.g., unwanted inclusions in the metal. They generally use visual assessment to identify the presence of these indications. Such indications can potentially occur at different stages in a multi-step production process.

Visual data analysis approaches that closely combine interactive data visualization with data analysis and processing methods are promising in many application fields, and these approaches are also of interest in the metal production industry. Appropriate methods in this area support the exploration and detection of patterns in large datasets, enabling, e.g., production engineers to identify opportunities for process improvements [1] [2].

In this paper, we present the results of a study on the design and application of a visual data analysis approach for the aluminum casting industry. This research was carried out in collaboration with a leading manufacturer of high-end aluminum products. A simplified aluminum casting production process from the recyclable material up to the final products is illustrated in Figure 1.

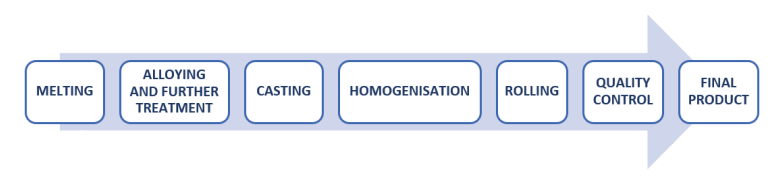

Figure 1. Schematic representation of process workflow

In a parallel aluminum casting process, several ingots are cast in each batch using a casting pit. Ingots are shapes that are cast from melted aluminum. These shapes are then suitable for fabrication processing by applying methods such as rolling, extrusion, and forging [3]. These ingots are rolled to form plates and sheets. Ultrasonic tests are performed to test the material quality of rolled aluminum plates to ensure that the final products meet high-quality standards. While different filtering elements are included in the production process, the final product may still show some indications e.g., unwanted inclusions. Ultrasonic testing is conducted to locate the position and size of such indications. The production process in the aluminum industry is time-consuming and can take up to several weeks, from the melting stage to the rolling stage and on to the production of the final product. If the final product does not meet the defined high-quality standards, the process must begin again, i.e., the product must be 
re-melted (Figure 1), resulting in extra production costs. During the production process analysis, explaining and eventually reducing indications are established as key priorities to meet high-quality standards [4].

To increase the product quality and production yield, it is necessary to identify the parameters which influence the product quality in aluminum casting. Engineers position sensors at various production steps which deliver a stream of production data. These data are time-dependent and typically multi-dimensional. While the data are continuously captured, the preprocessing and analytical steps are challenging due to the size and heterogeneity of the dataset. Also, data need to be linked appropriately. The main challenge that must be overcome, however, is to map the time-dependent production data to the run length of the cast aluminum. Researchers and industrial partners are actively searching for an effective way to visualize this complex data interactively. This visualization will allow users to explore the data and visually inspect them to identify the possible influences of various production parameters on product quality, promoting a better understanding of these parameters in production.

Our research group worked together with domain experts for several months in a project. While creating an iterative design process, we developed an interactive visual data analysis solution on the basis of the domain requirements obtained from a team of production experts. In this paper, we introduce this system which we have named ADAM [5] (an acronym for Aluminum production Data Analysis and Monitoring) and describe an extension to this system. The system was developed specifically to present a meaningful overview of and details about parameters relevant to the production process for the end users (i.e., material engineers in casting and rolling). ADAM provides an excellent tool that material engineers can use to explore data, rather than to make final interpretations. The data exploration using ADAM described in this study showed that an interactive data visualization tool can be used effectively to explore quality data, generate new hypotheses, and deepen the analysis. ADAM includes functions that allow the user to heuristically identify interesting portions of data, guiding the user's further analytical steps. The first system prototype was successfully integrated into the aluminum producer's system landscape and was tested for several months by material engineers. Below, we present our system design and discuss the advantages of and possible extensions to this system, including application examples to demonstrate the benefits of the tool and our findings.

\section{Related work}

Due to the digitization of industry and the multitude of novel data-driven applications possible (so-called Industry 4.0 or smart production), the use of data analysis and visualization in various areas - including manufacturing - plays a crucial role in continued growth, productivity, and innovation [6]. As the amount and complexity of production data grows, visual data analysis can be applied as an effective approach to gain insight into production data. Such analyses enable the users to interact actively with data and to explore and analyze data by the means of creating visual displays [7]. The increasing amount of data that has become available has driven the use of visualization tools and the development of visual data analysis tools in a variety of industrial domains [8]. However, many manufacturing systems have not been designed in ways that allow production specialists to efficiently and effectively analyze the steadily increasing amounts of heterogeneous production data. Appropriate analytical tools and interfaces are also lacking [9].

Few visual analysis solutions that target production scenarios have been presented in recent years. In one early study [10], the authors visualized sensor measurements and successfully applied information visualization techniques to process monitoring. Joe et al. [11] introduced a visual analysis tool that can be used to explore large manufacturing schedules. These authors extended the basic Gantt chart by adding novel algorithms and interactions. ViDX [12] is visual analytics system that supports the real-time tracking of assembly line performance and enables historical data exploration, demonstrating that visualizations can help domain experts detect outliers more effectively. Recently, Zhou et al. [13] published a survey on visualization and visual analysis applications for smart manufacturing. The survey reviews the findings of several studies conducted for industrial applications. Some examples of smart manufacturing applications used in the iron and steel industry are available. Production planning of metal ingot casting is specifically addressed in [14]. The latter work reports that visualization with interactive techniques will enhance advanced planning systems and improve usability. Zhou et al. [15] proposed the integration of advanced simulation and visualization methods for application in the manufacturing process, addressing the issues of energy, environment, productivity, safety, and quality in steel.

The development of our ADAM system has been partly inspired by work conducted on exploratory search methods [16] [17] and visualization tools and 
techniques [18]. Interactive visual analysis methods can be used to analyze complex and heterogeneous datasets [19]. Many interactive visualization systems are available, and we can only mention a selection here. Brigdan et al. [20] developed an interactive tool that can be employed for the investigation of medical data. The interactions include linked views and filters. The Spinel Explorer applies the basic principles of visual analysis and coordinated multiple views [21]. Voyager is a mixed-initiative system that couples faceted browsing with visualization recommendations to support exploration [22].

As our group focused on discovering the influence of individual parameters on production data, we noted that none of the existing tools met the specific requirements of the aluminum casting process and, therefore, none could have been employed directly. At the beginning of the project, we specifically studied how we could effectively perform the tasks of domain-dependent data extraction and transformation and derive suitable quality criteria for this production setting. The developed system ADAM is a tool that has several major strengths: It supports interactive filtering and zooming, provides multiple views through its linking and brushing functionalities, and enables both forward and backward searches to link quality data with production data.

\section{Visual Analytics Tool ADAM}

Traditionally, domain experts work with spreadsheet tools (i.e., MS Excel) to visualize, explore, and correlate data. In our case, these data were ingot production quality data. Excel was recognized as being suitable for viewing these data, performing the preliminary data analysis, and drawing basic graphs, but it was not suitable for the data cleaning step and more advanced data analysis tasks. When more complex data are included in a dataset, these data can only be processed manually in Excel if the data do not exceed certain size limits. If more parameters are included or the dataset is larger, it becomes challenging to extract significant information from the data. Conducting a simply baseline analysis does not allow the user to determine why certain cast ingots have higher or a lower quality or to identify the most influential parameters in the production process. However, if a statistical environment such as Python, R, MATLAB, or SAS is used, the code template allows for a much greater flexibility, enabling the user to manipulate data by applying data frames, arrays, and other functions. Prior to developing ADAM, we recognized that we needed to meet the key requirement to be able to study the data over a long period, select variables, and generally and quickly select parameters to compare these against inclusion measures. Hence, the review of the literature and our own personal observations indicated a strong need for techniques that the user can apply to discover and investigate the data more efficiently and effectively. From the beginning of ADAM's development, we had a goal to be able to monitor the distributions of indications in aluminum casts by analyzing a large set of different parameters. In the sections below, we introduce an appropriate visual interactive data analysis design present application examples, demonstrate the benefits of the use of the system, and discuss the findings.

\subsection{Dataset}

We collected the dataset used to develop ADAM from different data sources, starting from the input raw material to the final aluminum product. The quality dataset was obtained by conducting ultrasonic tests on cut aluminum plates with different lengths, widths, and thicknesses. These plates were produced from the cast and rolled ingots. Ultrasonic testing was used to locate the positions and sizes of indications on rolled aluminum plates. One of the biggest challenges faced was to match the indications that were detected on the final plates to the ingot length. Data were prepared with Pandas, one of the main tools used by data analysts, which is written in the programming language Python [23]. We then performed the first steps of data reduction, cleaning, and transformation. While carrying out these steps, we selected relevant data; handled incomplete data, missing values, and outliers; removed duplicates; and recalculated values from the final plates back to the original ingot.

\subsection{Design Process and Requirement Analysis}

Following an iterative design process under the guidance of a team of production experts, we formulated a set of domain requirements that enabled us to design the interactive visual data analysis solution. In this process, we focused on meeting the overall requirement to provide an overview of quality control in aluminum ingots.

R1 Use familiar visual elements and respect the users' mental models of aluminum casting. Since our target user group does not have experience with advanced visual designs, it was important for us to represent the ingot visualizations in an intuitive and easily understandable way. Therefore, together with target users, we selected specific visual elements to illustrate the parameters of the production process meaningfully and clearly. These elements were 


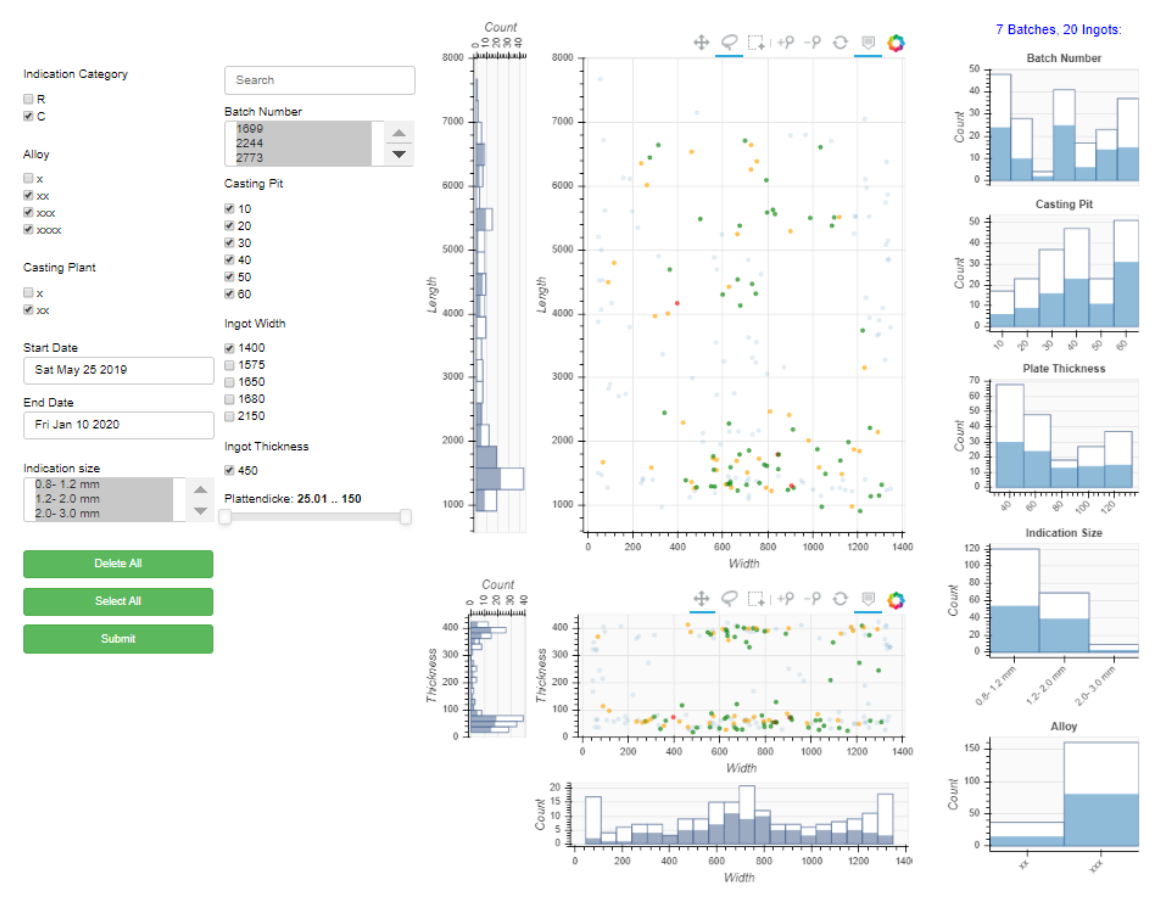

Figure 2. Visual analytics tool ADAM. Ingot visualization is composed of scatter plots and frequency histograms. Highlighted parts of the frequency histograms show the selected regions of points.

considered as possibly relevant for the quality of the final product. Two scatter plots were created to visualize the ingot, representing its front and top views. The axes of the scatter plots represent the length, width, and thickness, respectively.

To meet the final product requirements, we focused on exploring and comparing both single and multiple ingots and batches of ingots.

R2 Enable exploration and comparison of a single ingot as well as of several batches of ingots. Different parameters influence the quality of ingots and batches of ingots. To identify potential causes of indications in the production process as well as key influencing parameters, we needed to explore and compare the distributions of indications in single ingots, multiple ingots, and batches of ingots.

R3 Enable exploration and comparison of the distribution of indications for ingots in the same batch. In a parallel aluminum casting process, several ingots are cast in a casting pit in each batch. The quality of ingots in the same batch may vary and, in some extreme cases, variations from high to low quality may be seen. Domain tasks often include the exploration and comparison of ingots belonging to the same batch.
R4 Support filtering and selecting ingots or batches of interest. The visualization system was developed to support interactions among the filters and the selection of ingots and batches of interest for a specific distribution of indications and to present detailed information about the selected distributions. Some specific indication patterns only occur for certain ingots or batches, enabling a domain expert to investigate these further.

R5 Support exploration and detection of patterns with interaction techniques in multiple views. The casting length is directly associated with stages in the production process. Users should be able to interactively investigate atypical distributions of indications in the casting length by using the filtering, selecting, zooming, highlighting, linking, and brushing functions. Interactive changes made within one view are automatically reflected in all other views. If the user selects portions of data by applying the brushing and linking functionality in one view, this should only highlight selected points in the other view and avoid creating a new representation. 


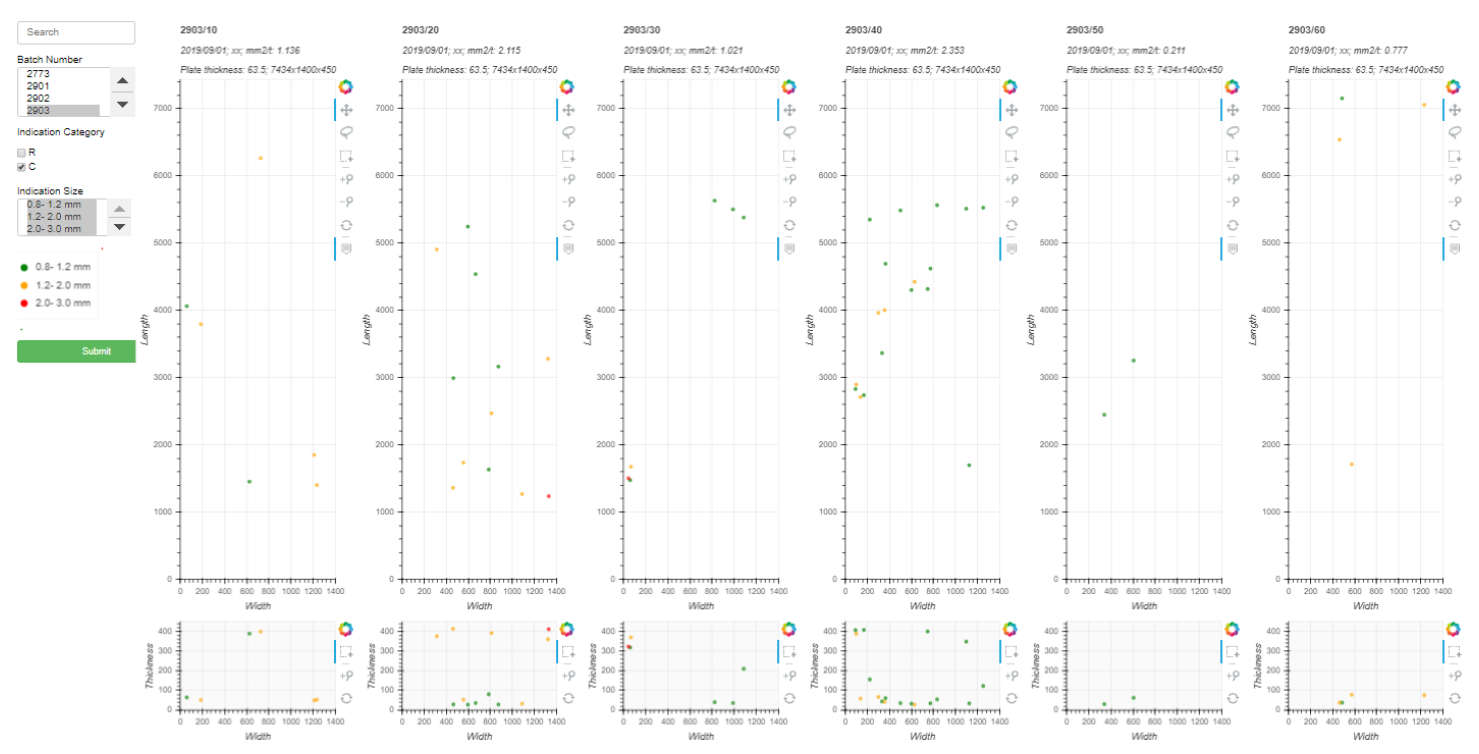

Figure 3. Additional detailed view representing ingot(front and top views)in the same batch. Domain tasks often include exploration and comparison of ingots belonging to the same batch.

\subsection{Visual Data Analysis Design}

The design of ADAM as our interactive visual data analysis tool is shown in Figure 2. The tool consists of two parts: (1) the visualization of ingots, which is composed of scatter plots and frequency histograms and (2) a set of filters and frequency histograms for the given filters.

Several workshops were carried out, and feedback was collected from domain experts. As a result, two scatter plots for the ingot visualization were selected. These plots show the intuitive front and top views of the distribution of indications. These views are also linked with three frequency histograms. Scatter plots, which are commonly used to visualize observed relationships between variables, use dots to represent values for two different numeric variables. In addition, histograms were selected as a visualization tool to provide information about the number of indications on the surfaces (i.e., length, width, and thickness) of each cast ingot. Color-coded circles shown in the scatter plots represent the values of indications with specific diameters. Green, orange, and red circles correspond to indications with the following sizes: $0.9-1.2$ $\mathrm{mm}, 1.2-2.0 \mathrm{~mm}$, and $2.0-3.0 \mathrm{~mm}$. The system includes filters such as batch number, casting pit, plate thickness, indication size, and alloy, and the user can apply these to explore and compare data interactively. The tool can be used effectively to examine single ingots, as well as examine and compare multiple ingots or several batches of ingots with one another. It was necessary to be able to explore the distribution of indications among ingots in the same batch. To meet this need, we extended the tool and provided the additional view represented in Figure 3. Interaction plays a significant role in visualization, especially when performing exploratory analysis tasks. If the analyst can make more selections, they will have more freedom to explore the data and potentially find relevant patterns. An example of the different interactions that can arise is shown in Figure 2. By applying the available interaction techniques, users can directly navigate and modify the visualizations, as well as select subsets of the data to carry out further operations [24]. All histograms in the tool are linked to scatter plots, and users can respond immediately to any selection. Hence, exploration is supported, and users can efficiently drill down within the data. Scatter plots support interactive visualization using the interaction techniques: pan, zoom in, box select, lasso select, and reset. Interactive changes made in one visualization are automatically reflected in all other visualizations. With the help of the brushing functionality, it is possible to select a region of points within one scatter plot, highlighting the parts with selected and non-selected points on the frequency histograms and in the other scatter plot. Highlighting parts on frequency histograms will enable the user to perform a backward search, i.e., extracting all casts that fall within the respective selected parameter bins. The forward and backward searches, when performed together, can yield unexpected findings. These findings can then be readily interpreted by domain users. For 
example, if the user runs a backward search to identify histograms of the selection that differ significantly from the histogram compared to all data, these differences may indicate that only certain batches, plate thicknesses, etc. would produce specific indication patterns. The domain expert would then be prompted to investigate these further. An example is shown in Figure2 (right). By selecting different parts of the cast data, users can immediately compare the selected distribution data with the full dataset, identify any interesting deviations, and subject these to a detailed inspection. In addition, the hover tool is added to scatter plots and histogram batch numbers. To obtain an informative view of the data, the user can apply the hover tool to a batch histogram to obtain information about a batch number and number of indications or apply it to the scatter plots to obtain information about batch numbers and casting pits.

Well-known business intelligence tools like Power BI and Tableau do not or only partially offer interaction techniques that are needed to meet domain requirements. Currently, Power BI does not allow user to perform scatter plot highlighting, although users do have the possibility to filter the dataset if edit interactions are desired. Power BI offers experienced web developers support in creating custom visuals. This tool is being continuously updated to respond to community suggestions and provide users with advanced features, which means that any custom visuals based on older versions are deprecated and must be frequently updated. The Tableau tool offers several methods for users to interact with data; one method that is relevant for meeting our requirements is the highlighting method select marks (tooltips). In this case, highlighting works well with small domains or views that contain a small amount of data. This tool, however, fails to completely satisfy our requirements with respect to larger datasets. Furthermore, if we highlight items in multiple views in Tableau, we need to use the master views method to filter the data. In addition, we are only able to highlight one value set at a time using the highlighter functionality in the tool. For this reason, we decided to develop a customized, responsive implementation for the target application.

Implementation ADAM was developed using Bokeh, a Python software library [25]. Bokeh enables the construction of information visualizations and extends this capability with strong interactivity functionality over potentially large or streaming datasets. This library was ideal for our purpose.

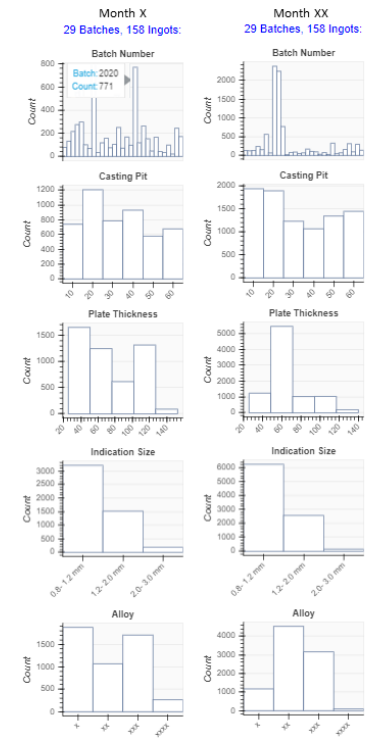

Figure 4. Comparison of histograms over two production months in different parameters.

\section{Results and Use Cases}

In this section, we present a discussion of the first results achieved by using ADAM, including examples to demonstrate the value of findings. The proposed visual analytics tool was tested by users in a real production environment. ADAM was used several times a week to monitor the distribution of indications as part of the quality control (Figure 1). We conducted interviews with material engineers involved in this study and report their evaluations of our approach.

\subsection{Data Exploration and Comparison}

ADAM could be used to meet the requirements to explore data for single ingots as well as explore and compare data collected from several batches of ingots and multiple ingots. Below, we illustrate several usage scenarios for data exploration and visual comparison. The proposed visual analytics tool allows users to explore data, providing an insight into data on various outcomes, and to investigate groups of multiple parameters. By applying different filters and using various interaction techniques, which are part of the ADAM system, we can easily select subsets and identify differences among subgroups.

The standard exploration process in ADAM consists of several steps. First, the user defines different filters based on their analytical interest. Next, the user explores indications in scatter plots and histograms. If the user notices an atypical distribution or patterns within data, they can select a region of points using the box select 
or lasso select function. This automatically highlights selected points on the scatter plots and histograms, as shown in Figure 2. Once parts of the frequency histograms have been highlighted, users can conduct a backward search. They can then search for a reverse selection, whereby the histograms of the selection differ significantly from the histogram generated from all data. Once the analysis has been completed, the user saves the results. These results can then be used for internal technical reporting.

While ADAM is primarily used to explore data, it can also be used to compare data. A typical analytical task is to monitor changes in the distribution of certain parameters over time, such as the batch number, casting pit, plate thickness, indication size, and used alloy. We note that the tested material is generally weighed in tons. A single cast ingot that measures $450 \times 1400 \times 7000 \mathrm{~mm}$ weighs around 12.3 tons. Figure 4 shows a comparison between two histograms for two production months with the same number of tested ingots. We want to investigate whether the number of indications correlates with a certain parameter. The user previously selected the dimensions of the ingot (i.e., width $1400 \mathrm{~mm}$, thickness $450 \mathrm{~mm}$ ) and the casting plant. The histograms show that the distributions are similar only in terms of one parameter: the indication size. Regarding the plate thickness in month $\mathrm{XX}$, the number of indications increased from $50 \mathrm{~mm}$ to $70 \mathrm{~mm}$. This finding may indicate that only some batches, plate thicknesses, etc. show specific patterns of indications, suggesting that the domain expert should further investigate these batches. By applying the hover tool, however, we can determine the highest number of indications seen in this batch number in month $\mathrm{X}$. In month $\mathrm{XX}$, we also see that only a few batches have poor quality. Furthermore, the user can perform a backward analysis, excluding batches with poor quality and comparing the histograms again. These actions may provide different results.

\subsection{Analyzing Patterns}

Different groups of parameters influence the quality of batches and ingots, indicating that only some batches, ingots, plate thicknesses, etc. might have specific indication patterns. To discover possible causes of these indications in the production process and the associated key influencing parameters, we need to explore the distributions of indications in the batches of ingots and on the individual ingots.

Data exploration helps us to discover patterns in casting length. The casting length is directly associated with the production stage. The longer the ingot, the further along we are in the process of casting. This

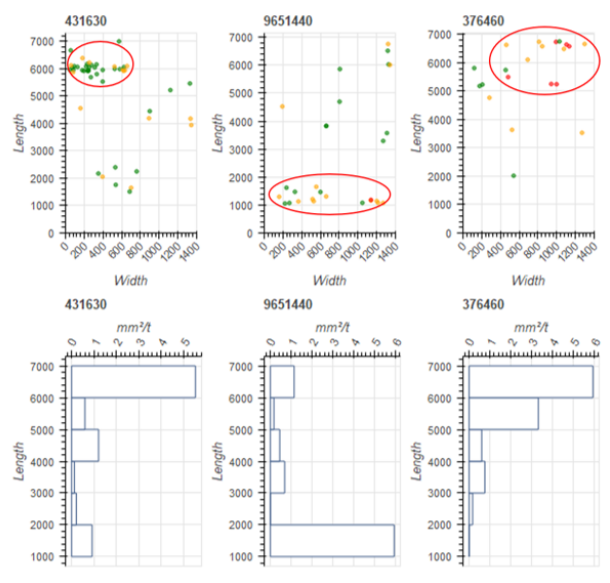

Figure 5. Patterns identified during analysis. Three visually prominent patterns (from left to right): 1) group of indications at a specific location on the ingot, 2) group of indications seen at the beginning of the casting, 3) group of indications seen at the end of the casting

means that we could investigate parameters that are influential at the beginning of the casting process or at the end of the casting process by identifying the position of the indications along the length of the ingot.

Production experts can use ADAM to quickly detect patterns in data, immediately recognize the behavior of these patterns, and find tendencies. Figure 5 shows visually identifiable patterns regarding the casting length. If the material is not free of indications, most of indications generally appear at the beginning of the casting length, and the number of indications decreases towards the end of the length. Using ADAM to explore the available data, production experts noticed that atypical distributions appear in some batches and on some ingots. In addition, they were able to further analyze the data and to associate certain production parameters from the casting with these indications in some cases.

Although ADAM allows users to explore data and avoid making incorrect interpretations, we need to take into consideration the fact that production datasets are often unbalanced, as real-world data are often skewed.

\subsection{Evaluation Results}

To evaluate our approach, we interviewed two domain experts who represented the target user group. Both domain experts stated that they used ADAM several times a week for exploratory data analysis and for internal technical reporting. However, they did not use the system for classic reporting. They agreed 
that one of the biggest advantages of ADAM is that it supports the rapid and easy visualization of large datasets. They also mentioned that it is very useful for extracting information from datasets, as it allows the user to select areas on histograms using different interaction techniques (e.g., box select, lasso selection, zoom in). They found it particularly useful that ADAM enables users to view the results for the whole batch as well as for single ingots. Domain experts reported that using ADAM enabled them collect data that they could then explore to identify special patterns, distributions of indications, and correlate these distributions with the process data.

Although the tool allows excellent data exploration, we also noted that the current system version has some limitations. The first domain expert stated that the tool could be improved by offering the possibility to identify outliers. Outliers can distort the results, as seen in the example discussed in the previous section. The second domain expert said that the data filtering function in the ingot view required certain further improvements and provided several helpful suggestions. This expert also noted that having templates for frequently used data selections would be helpful, such as previously selected ingot dimensions and casting plants. Both domain experts noted that the tool currently lacks a function for the automatic detection of repetitive patterns. Based on the results of these interviews, we are planning further improvements to ADAM as part of future work.

\section{Conclusions and Future Work}

We developed and introduced ADAM, an interactive visual data analysis tool, to support production data exploration in aluminum casting. Interactive data visualization tools such as this can be used to explore quality data, generate new hypotheses, and guide further analysis. ADAM supports interactive data production analysis and enables users to search for dependencies and patterns in data. In addition, we demonstrated the usefulness of ADAM and illustrate the insights that can be obtained by its application to real production data.

Future work includes extending ADAM so that it can carry out automated data exploration tasks, e.g., automatically suggest similar batches and ingots of interest, as well as batches and ingots with atypical distributions, to the user. Users need to be able to group similar batches and ingots in order to investigate the influence of specific production parameters more precisely. In future research, we will also consider the glyph-based visualization of multi-dimensional data. We are currently working to include specialized multidimensional data visualizations in ADAM to support pattern detection and correlating parameters. Furthermore, we have interest in extending ADAM in the future to enable the automatic classification of certain quality patterns, based on the interactively provided examples from experts.

Acknowledgement This research work is done by Pro2Future and AMAG Austria Metall AG. Pro2Future is funded within the Austrian COMET Program-Competence Centers for Excellent Technologies under the auspices of the Austrian Federal Ministry of Transport, Innovation and Technology, the Austrian Federal Ministry for Digital and Economic Affairs and of the Provinces of Upper Austria and Styria. COMET is managed by the Austrian Research Promotion Agency FFG.

\section{References}

[1] J. Suschnigg, F. Ziessler, M. Brillinger, M. Vukovic, J. Mangler, T. Schreck, and S. Thalmann, "Industrial production process improvement by a process engine visual analytics dashboard," in Proceedings of the 53rd Hawaii International Conference on System Sciences, pp. 1320-1329, 2020.

[2] D. Sun, R. Huang, Y. Chen, Y. Wang, J. Zeng, M. Yuan, T.-C. Pong, and H. Qu, "Planningvis: A visual analytics approach to production planning in smart factories," IEEE transactions on visualization and computer graphics, vol. 26, no. 1, pp. 579-589, 2019.

[3] A. K. Vasudevan and R. D. Doherty, Aluminum Alloys-Contemporary Research and Applications: Contemporary Research and Applications. Elsevier, 2012.

[4] B. Prillhofer, H. Antrekowitsch, H. Bottcher, and P. Enright, "Nonmetallic inclusions in the secondary aluminium industry for the production of aerospace alloys," in LIGHT METALS-WARRENDALE-PROCEEDINGS-, vol. 2008, p. 603, TMS, 2008.

[5] N. Jekic, B. Mutlu, M. Faschang, S. Neubert, S. Thalmann, and T. Schreck, "Visual analysis of aluminum production data with tightly linked views.," in EuroVis (Posters), pp. 49-51, 2019.

[6] J. Lee, H.-A. Kao, S. Yang, et al., "Service innovation and smart analytics for industry 4.0 and big data environment," Procedia Cirp, vol. 16, no. 1, pp. 3-8, 2014.

[7] W. Wu, Y. Zheng, K. Chen, X. Wang, and N. Cao, "A visual analytics approach for equipment condition monitoring in smart factories of process industry," in 2018 IEEE Pacific Visualization Symposium (PacificVis), pp. 140-149, IEEE, 2018.

[8] S. Liu, W. Cui, Y. Wu, and M. Liu, "A survey on information visualization: recent advances and challenges," The Visual Computer, vol. 30, no. 12, pp. 1373-1393, 2014.

[9] J. Lee, E. Lapira, B. Bagheri, and H.-a. Kao, "Recent advances and trends in predictive manufacturing systems in big data environment," Manufacturing letters, vol. 1, no. 1 , pp. 38-41, 2013. 
[10] K. Matkovic, H. Hauser, R. Sainitzer, and M. E. Groller, "Process visualization with levels of detail," in IEEE Symposium on Information Visualization, 2002. INFOVIS 2002., pp. 67-70, IEEE, 2002.

[11] J. Jo, J. Huh, J. Park, B. Kim, and J. Seo, "Livegantt: Interactively visualizing a large manufacturing schedule," IEEE transactions on visualization and computer graphics, vol. 20, no. 12, pp. 2329-2338, 2014.

[12] P. Xu, H. Mei, L. Ren, and W. Chen, "Vidx: Visual diagnostics of assembly line performance in smart factories," IEEE transactions on visualization and computer graphics, vol. 23, no. 1, pp. 291-300, 2017.

[13] F. Zhou, X. Lin, C. Liu, Y. Zhao, P. Xu, L. Ren, T. Xue, and L. Ren, "A survey of visualization for smart manufacturing," Journal of Visualization, vol. 22, no. 2, pp. 419-435, 2019.

[14] P. Y. Wu, "Visualizing capacity and load in production planning," in Proceedings Fifth International Conference on Information Visualisation, pp. 357-360, IEEE, 2001.

[15] C. Zhou, J. Wang, G. Tang, J. Moreland, D. Fu, and $\mathrm{B}$. Wu, "Integration of advanced simulation and visualization for manufacturing process optimization," Jom, vol. 68, no. 5, pp. 1363-1369, 2016.

[16] G. Marchionini, "Exploratory search: from finding to understanding," Communications of the ACM, vol. 49, no. 4, pp. 41-46, 2006.

[17] R. W. White and R. A. Roth, "Exploratory search: Beyond the query-response paradigm," Synthesis lectures on information concepts, retrieval, and services, vol. 1, no. 1, pp. 1-98, 2009.

[18] D. Keim, J. Kohlhammer, G. Ellis, and F. Mansmann, "Mastering the information age: solving problems with visual analytics," 2010.

[19] B. Shneiderman, "Inventing discovery tools: combining information visualization with data mining," Information visualization, vol. 1, no. 1, pp. 5-12, 2002.

[20] M. Brigdan, M. D. Hill, A. Jagdev, and N. Kamal, "Novel interactive data visualization: exploration of the escape trial (endovascular treatment for small core and anterior circulation proximal occlusion with emphasis on minimizing ct to recanalization times) data," Stroke, vol. 49, no. 1, pp. 193-196, 2018.

[21] M. L. Ganuza, G. Ferracutti, M. F. Gargiulo, S. M. Castro, E. Bjerg, E. Gröller, and K. Matković, "The spinel explorer-interactive visual analysis of spinel group minerals," IEEE transactions on visualization and computer graphics, vol. 20, no. 12, pp. 1913-1922, 2014.

[22] K. Wongsuphasawat, D. Moritz, A. Anand, J. Mackinlay, B. Howe, and J. Heer, "Voyager: Exploratory analysis via faceted browsing of visualization recommendations," IEEE transactions on visualization and computer graphics, vol. 22, no. 1, pp. 649-658, 2016.

[23] W. McKinney, Python for data analysis: Data wrangling with Pandas, NumPy, and IPython. "O'Reilly Media, Inc.”, 2012.

[24] D. A. Keim and M. O. Ward, "Visual data mining techniques," 2002.

[25] Bokeh Development Team, Bokeh: Python library for interactive visualization, 2018. 